\title{
(2) OPEN ACCESS \\ Dietary macronutrients and the gut microbiome: a precision nutrition approach to improve cardiometabolic health
}

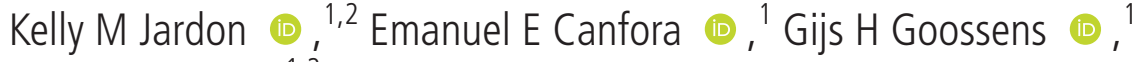 \\ Ellen E Blaak (1) 1,2
}

Human Biology, School of Nutrition and Translational Research in Metabolism (NUTRIM), Maastricht University Medical Center+, Maastricht, The Netherlands ${ }^{2} \mathrm{TiFN}$, Wageningen, The Netherlands

Correspondence to Professor Ellen E Blaak, Human Biology, School of Nutrition and Translational Research in Metabolism (NUTRIM), Maastricht University Medical Center+, P.O.Box 616, 6200 MD Maastricht, The Netherlands; e.blaak@maastrichtuniversity.nl

Received 26 November 2020 Accepted 17 January 2022
Check for updates

(C) Author(s) (or their employer(s)) 2022. Re-use permitted under CC BY-NC. No commercial re-use. See rights and permissions. Published by BMJ.

To cite: Jardon $\mathrm{KM}$, Canfora EE, Goossens GH, et al. Gut Epub ahead of print: [please include Day Month Year]. doi:10.1136/ gutinl-2020-323715

\begin{abstract}
Accumulating evidence indicates that the gut microbiome is an important regulator of body weight, glucose and lipid metabolism, and inflammatory processes, and may thereby play a key role in the aetiology of obesity, insulin resistance and type 2 diabetes. Interindividual responsiveness to specific dietary interventions may be partially determined by differences in baseline gut microbiota composition and functionality between individuals with distinct metabolic phenotypes. However, the relationship between an individual's diet, gut microbiome and host metabolic phenotype is multidirectional and complex, yielding a challenge for practical implementation of targeted dietary guidelines. In this review, we discuss the latest research describing interactions between dietary composition, the gut microbiome and host metabolism. Furthermore, we describe how this knowledge can be integrated to develop precision-based nutritional strategies to improve bodyweight control and metabolic health in humans. Specifically, we will address that (1) insight in the role of the baseline gut microbial and metabolic phenotype in dietary intervention response may provide leads for precision-based nutritional strategies; that (2) the balance between carbohydrate and protein fermentation by the gut microbiota, as well as the site of fermentation in the colon, seems important determinants of host metabolism; and that (3) 'big data', including multiple omics and advanced modelling, are of undeniable importance in predicting (non-)response to dietary interventions. Clearly, detailed metabolic and microbial phenotyping in humans is necessary to better understand the link between diet, the gut microbiome and host metabolism, which is required to develop targeted dietary strategies and guidelines for different subgroups of the population.
\end{abstract}

\section{INTRODUCTION}

The global rise in the prevalence of obesity is a major socioeconomic burden and is strongly associated with an increase in the incidence and prevalence of cardiometabolic diseases, including insulin resistance (IR) and type 2 diabetes mellitus (T2DM). ${ }^{12}$ Despite efforts to improve lifestyle choices and to increase insight in the underlying aetiology, long-term success in the prevention and treatment of obesity seems limited, as diet-induced weight loss is only maintained for approximately $25 \%$ after a 5 -year follow-up. ${ }^{3}$ In recent years, advancements have been made in understanding

\section{Key messages}

What is already known about this subject?

- The interaction between the gut microbiome diet and the host's metabolism is multidirectional and complex, providing a challenge in acquiring detailed, mechanistic insights.

- Gut-derived metabolites, including short-chain fatty acids (derived from fibre fermentation), may be important regulators of host metabolism. Most metabolites derived from protein fermentation seem to have detrimental effects on metabolic health.

What are new findings in this field and how could they impact future research and clinical practice?

- The baseline gut microbial profile may be a predictor for an individual's response to dietary interventions. Future research in this field should consider detailed characterisation of both microbial and metabolic phenotypes as well as their interaction.

- Gut microbiome-associated effects on host metabolism may be related to fermentation products of carbohydrates and proteins. Understanding how to optimally balance proteolytic and saccharolytic fermentation and gaining insight into the importance of the site of colonic fermentation will gain insight into the interplay between diet, gut microbiome and metabolic processes.

- Understanding the mechanisms of the differential responses to diet is essential to move forward in the field of precision nutrition. Although both the amount and quality of knowledge have evolved rapidly in recent years, we still only see the tip of the iceberg.

the involvement of the gut microbiome in obesity and related cardiometabolic complications as regulator of host energy and substrate metabolism. ${ }^{45}$ Targeting host metabolism via the gut microbiome may therefore be a putative strategy to improve the effectiveness of lifestyle interventions to promote cardiometabolic health.

The complexity of the gut microbiome itself and its interplay with the host's health and host energy and substrate metabolism, as well its role 
in obesity and T2DM, have become more evident. ${ }^{4-8}$ Despite an exponential increase in the number of studies investigating the impact of the gut microbiome on host metabolism over the past decade, data confirming a causal role of the gut microbiome in human metabolism are scarce and results are inconsistent. ${ }^{79}$ Moreover, findings of animal studies appear difficult to translate to humans due to differences in anatomy, genetics, physiology and gut microbiome-host interaction, among other factors. ${ }^{10} 11$ Throughout the 20th century, microbiome-host interactions have already gained scientific interest, ${ }^{12}$ providing the basis for human faecal transplant studies, which hint towards a causal role of the gut microbiota composition and its derived metabolites in the development of the metabolic syndrome. ${ }^{13} 14$ These studies have shown that baseline microbial composition may predict the improvement in peripheral insulin sensitivity in individuals with the metabolic syndrome, although effects were transient. ${ }^{14}$ Nevertheless, recently it has been demonstrated that strong alterations of the abundance of microbes induced by antibiotics had no significant effects on insulin sensitivity, inflammatory profile and energy/substrate metabolism in individuals with obesity and impaired glucose metabolism. ${ }^{15}$ This doubleblind, randomised, placebo-controlled clinical trial indicates that modulation of microbiota composition may not have an important impact on human metabolism under all conditions, and that this impact rather depends on the individual's characteristics such as age, habitual diet, metabolic phenotype and baseline gut microbial profile.

The composition of the gut microbiota is determined by hereditable, demographic and environmental factors, including mode of delivery at birth, age, sex, gastrointestinal transit time and use of medication. In recent studies, diet has emerged as a significant contributor to the shaping and defining of the gut microbiome. ${ }^{16-19}$ Diet-induced changes in the composition and functionality of the gut microbiota have been linked to the development of obesity and related disorders. ${ }^{20}$ Dietary fibres, in particular, have received great attention as potential mediators of the host's metabolism via gut microbiome-related interactions, ${ }^{21}$ but conflicting findings have been reported regarding metabolic outcomes. ${ }^{22}{ }^{23}$ As indicated previously, differences in baseline gut microbiota composition and functionality may determine the (lack of) response to an intervention. ${ }^{24}$ As such, it is tempting to postulate that interindividual variation in gut microbiota composition and functionality may serve as a basis for a more precision-based dietary approach in counteracting cardiometabolic diseases.

In this review, the complex interaction between dietary macronutrients, in particular dietary fibres and proteins, gut microbiome and host metabolic health, is addressed. The present review focusses on gut microbial metabolites produced in saccharolytic fermentation such as short-chain fatty acids (SCFAs) as well as proteolytic fermentation like ammonia, indolic and phenolic compounds, and branched-chain fatty acids (BCFAs) (figure 1). We will discuss the balance of saccharolytic and proteolytic fermentation as important determinant of metabolic health. It is increasingly evident that this balance may be modulated by diet in a person-specific or subgroup-specific manner. ${ }^{25}$ At first, it will be briefly addressed to what extent microbial composition and its functionality relates to the host's metabolic phenotype, including the putative underlying mechanisms related to gutderived saccharolytic and proteolytic metabolites. Subsequently, we will discuss the impact of dietary fibres and protein on microbial composition and functionality and insulin sensitivity, as well as to what extent response and non-response are mediated by baseline microbial composition. Overall, we will provide insight into the importance of precision nutrition strategies to modulate microbial composition and functionality and, thereby, bodyweight control and insulin sensitivity. Finally, we will address what is required to translate findings within this field into more precision nutrition-based interventions and guidelines.

\section{GUT MICROBIOME AND HOST METABOLIC PHENOTYPE Gut microbial composition in obesity and T2DM}

The gut microbiome seems to impact the host's metabolic phenotype through the production of different metabolites. ${ }^{26}$ Through these metabolites, the gut microbiota may affect immune function and epithelial integrity, energy and substrate metabolism, and glucose homeostasis. ${ }^{13} 2728$ This capability of interacting with host health is dependent on the type of bacteria present and the distribution of these bacteria in the gut microbial community. Since functionality (eg, fermentation capacity) varies among bacterial species, alterations in gut microbiota composition and diversity, as observed in obesity and T2DM, may subsequently affect metabolic processes and the immune system of the host.

The core human gut microbiota mainly consists of the bacterial phyla Firmicutes, Bacteroidetes, Actinobacteria, Proteobacteria, Fusobacteria and Verrucomicrobia, of which Firmicutes and Bacteroidetes represent approximately $90 \%$ of the gut microbiota community. ${ }^{29}$ Ninety-five per cent of the Firmicutes phylum is represented by Lactobacillus, Bacillus, Clostridium, Enterococcus and Ruminococcus genera, while Bacteroidetes predominantly consists of Bacteroides, Prevotella and, to a lesser extent, Actinobacteria, which includes the Bifidobacterium genus. ${ }^{29}$ Although a core microbiota exists, its composition is highly individualised and shaped by genetics, ${ }^{30}$ sex, ${ }^{31} 32$ age, ${ }^{33} 34$ ethnicity ${ }^{35}$ and environmental factors, such as drug use ${ }^{2836}$ and habitual diet. ${ }^{37}$ The uniqueness of the human gut microbiota profile translates into highly individualised responses to acute dietary challenges and longer-term dietary interventions. ${ }^{38-40}$ The success of an intervention might therefore be at least partially explained by baseline differences in the gut microbiome.

Interestingly, several studies demonstrated a relationship between the metabolic phenotype of an individual and the composition and functionality of the gut microbiota. Initial studies indicated an increased Firmicutes-to-Bacteroidetes ratio in humans and rodents with obesity as compared with lean individuals, ${ }^{41}{ }^{42}$ but other studies have failed to observe such a difference or even reported a decreased ratio. ${ }^{94} 44$ More consistent, overweight and moderate obesity, IR and T2DM have been associated with compositional changes and decreased microbial richness and diversity when compared with lean, healthy individuals, ${ }^{4145-47}$ and this has been linked to metabolic impairments. Indeed, detailed characterisation of individuals with overweight or (morbid) obesity showed that microbial gene richness is inversely correlated with fat mass, leptin levels, fasting insulin, homeostatic model assessment of insulin resistance (HOMA-IR), triglyceride levels and systemic inflammation. ${ }^{45}$ The prevalence of low microbial gene richness was found to be as high as $75 \%$ in severe obesity, compared with $23 \%-40 \%$ in lean or overweight/ moderate obesity. ${ }^{48}$ In a comparison of metabolically healthy versus unhealthy individuals, alpha diversity was lower in the metabolically unhealthy group. ${ }^{49}$ Specifically, bacteria of the Oscillospira genus and Coriobacteriaceae family were associated with good metabolic health. In a study population encompassing people with normal weight and overweight/obesity, the abundance of specific genera correlated with metabolic characteristics. ${ }^{50}$ Notably, anthropometric parameters were positively associated with Collinsella aerofaciens, Dorea formicigenerans 

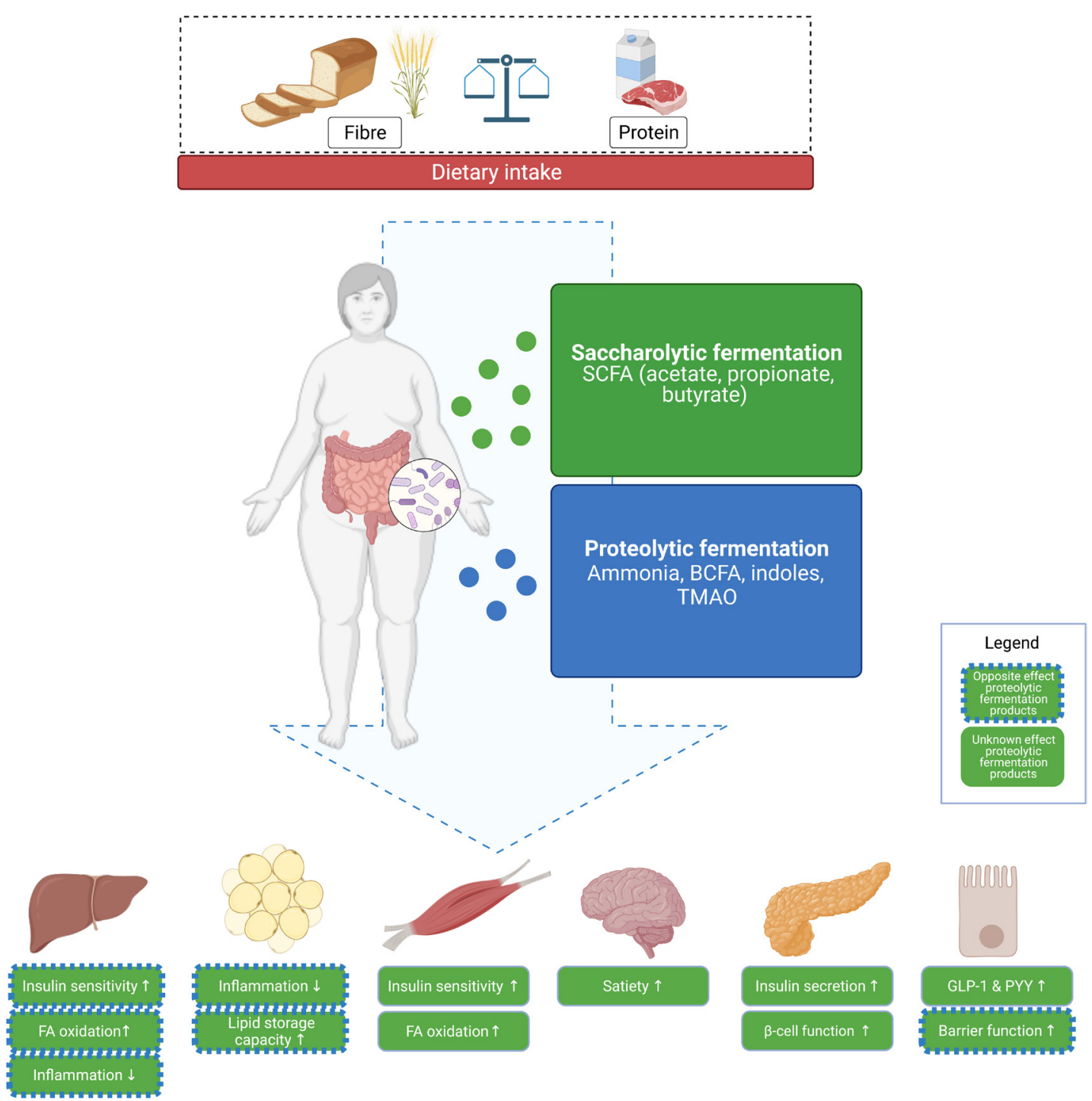

Figure 1 Interactions between diet and saccharolytic and proteolytic fermentation in the gut and host metabolism. Fermentation of dietary fibres occurs mainly in the proximal colon and yields SCFAs that can both be used as fuel for enterocytes and can act as peripheral signalling molecules. SCFAs are involved in centrally regulating food intake and energy expenditure by effects on secretion of GLP-1 and PYY. SCFAs are beneficial regulators of interorgan crosstalk between the gut and peripheral organs like the liver and muscle. Protein fermentation mainly occurs in the distal colon and yields a more diverse range of metabolites, including BCFAs, which are associated with detrimental effects on gut and metabolic health. Green boxes indicate effects of SCFAs on metabolic processes in peripheral organs. Blue borders indicate effects in the opposite direction site direction (dotted line) or unknown direction (no line) of proteolytic fermentation products. BCFA, branched-chain fatty acid; FA, fatty acid; GLP-1, glucagon-like peptide 1; PYY, peptide YY; SCFA, short-chain fatty acid; TMAO, trimethylamine N-oxide.

and D. longicatena, which had higher abundance in people with overweight/obesity. Moreover, different genera of Lachnospiraceae were negatively associated with body fat mass, low-density lipoprotein and total cholesterol concentrations. The involvement of individual genera or species in metabolic regulation seems to be confirmed by numerous other, mainly association studies in humans. Most compelling evidence is available for bacteria of Akkermansia genus, which is negatively associated with the risk of developing obesity and metabolic syndrome. ${ }^{5152}$ A 3-month supplementation of Akkermansia muciniphila improved insulin sensitivity and reduced blood markers of liver dysfunction and inflammation in individuals with IR and overweight/obesity. Furthermore, another study showed significant enrichment of both A. muciniphila and Alistipes obesi in lean individuals, and of Ruminococcus gnavus in obese individuals. A 6-month calorie restriction diet increased $A$. muciniphila and $A$. obesi even more in lean individuals, while $R$. gnavus abundance decreased in the obese group. ${ }^{53}$ This study identified Blautia wexlerae and Bacteroides dorei as strongest predictors for weight loss when present in high abundance at baseline. Specific interventions with highfibre diets, however, hint towards baseline Prevotella abundance as predictor for weight loss success on these diets in people with obesity. $^{54}$

Additionally, patients with T2DM and metabolically compromised individuals showed an altered microbial functionality and a decreased fermentation capacity when compared with healthy individuals with, in particular, a lower abundance of butyrateproducing bacteria. ${ }^{135556}$ Furthermore, the gut microbiome of insulin-resistant individuals may have increased biosynthesis potential and decreased uptake and catabolism of branchedchain amino acids (BCAAs, largely driven by Prevotella copri and B. vulgatus), which have been linked to detrimental metabolic 
effects. ${ }^{57}$ Taken together, there is evidence for decreased microbial gene richness and diversity in metabolically compromised individuals. Moreover, specific individual microbes may be regulators in (changes in) cardiometabolic health. Interindividual differences in gut microbiota composition and functionality may be linked to an altered responsiveness to (dietary) interventions, as will be discussed more extensively later in this review.

\section{Saccharolytic and proteolytic fermentation, bodyweight control and insulin sensitivity}

The interaction between the gut microbiota and host metabolism is multifactorial and depends on the microbial and host metabolic phenotype, which may be strongly inter-related. The production or activation of signalling molecules involved in host metabolism, the regulation of bile acid homeostasis, modification of gut permeability, the release of gut hormones, lipopolysaccharides and inflammatory markers, are among the mechanisms by which gut microbiota may influence the host cardiometabolic phenotype. ${ }^{58}$ Gut-derived metabolites may be either beneficial or detrimental to host metabolic health. In developing precision-based nutrition, the capacity of bacteria to produce certain metabolites should be considered. In this review, we focus on the balance between saccharolytic and proteolytic fermentations as a determinant of metabolic health (figure 1), as will be discussed in the following paragraphs.

\section{Saccharolytic fermentation}

The gut microbiota is capable of fermenting food components that otherwise would be unavailable to the host. ${ }^{59}$ Dietary fibres and other complex carbohydrates that cannot be broken down by enzymes in the small intestine can (partly) be fermented by bacteria in the large intestine as preferred energy source, producing microbial products such as SCFAs (predominantly acetate, propionate and butyrate) ${ }^{60}$ The main butyrate producing bacteria belong to the phylum Firmicutes, in particular Faecalibacterium prausnitzii, Clostridium leptum, Eubacterium rectale and Roseburia spp. ${ }^{61}$ The production of other SCFAs is mediated by bacteria such as Bifidobacterium that produce acetate and lactate during carbohydrate fermentation. Also, A. muciniphila species produce both propionate and acetate. ${ }^{61} 62$

Stable-isotope techniques with $13 \mathrm{C}$ labelled SCFAs allow quantification of in vivo colonic production of SCFAs based on breath, urine and blood analyses. ${ }^{63}$ SCFAs are formed primarily in the colon, in which approximately $95 \%$ are subsequently absorbed. ${ }^{64}$ Butyrate is largely used as the major energy source by colonocytes, while propionate and acetate travel to the liver via the portal vein. In particular, acetate can also reach the peripheral tissues after entering the systemic circulation, inducing a diversity of metabolic and satiety-related effects. ${ }^{25} 65$ SCFAs can bind to G protein-coupled receptors (GPRs). The best studied receptors include GPR41, GPR43, GPR109a and GPR164, which are expressed in a vast array of cells, including the colonic epithelium, pancreatic $\beta$ cells, immune cells and peripheral tissues, like adipose tissue. ${ }^{66}$ Effects of SCFAs on peripheral tissues include increased adipogenesis, inhibition of adipose tissue lipolysis (specifically by acetate) and attenuation of inflammation in adipocytes, ${ }^{6567-69}$ increased lipid oxidative capacity in skeletal muscle, ${ }^{70} 71$ increased pancreatic insulin secretion and $\beta$-cell function, ${ }^{72} 73$ increased insulin sensitivity and lipid oxidation in the liver ${ }^{7475}$ and altered gut-brain interaction. ${ }^{76-79}$ These data are mainly derived from in vitro and rodent studies.

In human studies, however, data on associations between SCFAs and metabolic parameters cannot always be reproduced.
For example, plasma insulin levels increased during hyperglycaemic clamps with acetate infusion independently of changes in plasma acetate in obese individuals. ${ }^{80}$ Beneficial effects of SCFAs may also be related to an increased secretion of intestinal incretins, such as glucagon-like peptide 1 (GLP-1) and peptide YY (PYY). Acute colonic administration of propionate increased postprandial plasma PYY and GLP-1 and reduced energy intake. ${ }^{81}$ Furthermore, long-term colonic propionate delivery prevented bodyweight gain, reduced abdominal obesity and intrahepatocellular lipid content, and prevented the deterioration in insulin sensitivity in overweight adults. ${ }^{81}$ In line with these findings, human in vivo data indicated that in adults with overweight or obesity, fasting lipid oxidation and resting energy expenditure increased after diet-induced changes in microbial SCFA production or direct colonic SCFA infusions. ${ }^{25}$ 82-85 Although most studies indicate a beneficial role for SCFAs in energy and glucose homeostasis, ${ }^{658687}$ larger human studies are needed to elucidate the complexity of underlying mechanisms.

\section{Proteolytic fermentation}

In a population consuming a Western diet low in dietary fibres, the microbiota of the distal part of the colon are more specialised in fermentation of residual peptides and proteins ${ }^{25}$ because the preferred fuel, fermentable carbohydrates, is already largely used in the proximal colon. ${ }^{88}$ Products of this proteolytic fermentation process include gaseous products such as hydrogen, methane, carbon dioxide and hydrogen sulfide; BCFAs isobutyrate, 2-methylbutyrate and isovalerate derived from fermentation of BCAAs, phenolic and indolic compounds derived from microbial fermentation of aromatic amino acids and to a lesser, unknown extent SCFAs. ${ }^{89}$ In contrast to saccharolytic fermentation products, most products derived from proteolytic fermentation are considered detrimental for host gut and metabolic health, ${ }^{60} 8990$ although some data in animals suggest a beneficial role of indole and hydrogen sulfide in gut and peripheral tissue function. ${ }^{91} 92$

The balance between saccharolytic and proteolytic fermentation in the colon, and thus the balance between putatively beneficial and detrimental regulation of the host's physiology, may be of interest in the development of dietary intervention strategies. Some studies showed that an increased dietary fibre intake, in particular intake of slowly fermentable fibres, reduces the production of merely detrimental proteolytic metabolites by the gut microbiota, ${ }^{24} 93$ suggesting a shift in overall fermentation balance towards the more beneficial saccharolytic fermentation.

Additionally, dietary choline and carnitine derived from, for example, red meat can be converted into trimethylamine (TMA) by microbial enzymes in the gut. This TMA reaches the liver via absorption and transportation via the portal vein. In the liver, TMA can be oxidised by hepatic flavin-containing monooxygenase into trimethylamine $\mathrm{N}$-oxide (TMAO), which is strongly associated with the development of cardiovascular diseases, non-alcoholic fatty liver disease and systemic inflammation. ${ }^{94-96}$ Furthermore, in both murine and human studies, evidence is increasing that TMAO also contributes to the development of IR and T2DM. ${ }^{97-99}$ Increasing the availability of dietary fibres to the whole colonic microbiota might decrease the fermentation of dietary choline and carnitine, and therefore inhibit the production of the TMAO precursor TMA. Indeed, a rodent study demonstrated that a high amount of fermentable fibres derived from wheat bran lowered colonic TMA lyase activity. ${ }^{100}$ However, whether this can be translated to humans has to be elucidated. 


\section{DIETARY MACRONUTRIENTS, MICROBIAL COMPOSITION AND METABOLIC HEALTH}

Shaping of the adult gut microbiome is already initiated in early life, depending on factors such as exposure to the maternal microbiome, the mode of delivery and early exposure to dietary components, ${ }^{101}$ although the contribution of these factors cannot always be confirmed. ${ }^{102}$ Of the many factors influencing gut microbiota composition and function throughout all life stages, diet is key in modulating abundances of specific bacterial species and their functions. ${ }^{16} 103$ Vice versa, an individual's response to a certain diet or dietary components may be largely influenced by features of the gut microbiota. ${ }^{39}$ This bidirectional interaction between diet and an individual's microbiota forms a basis for understanding the concept of precision nutrition in the light of a third component: the host's metabolic health.

\section{Diet, macronutrients and microbial profile}

In the current Western world, habitual diet composition has shifted towards a diet high in energy-dense foods, including relatively high amounts of saturated fat and simple carbohydrates, and low amounts of dietary fibre. Especially the consumption of dietary fibre, but also the macronutrient quality along with the quantity consumed can strongly influence gut microbiota composition and functionality. Population-based metagenomics analysis revealed associations between microbial composition and diversity and the habitual diet for over 60 dietary factors. These factors include energy and macronutrient intake and consumption of specific food items such as bread and soft drinks. These data confirm the importance of diet in shaping the gut microbiome. ${ }^{37} 104$

In a longitudinal monozygotic twin study, stool microbiota analysis showed that habitual intakes of energy, type of unsaturated fatty acid (FA) and soluble fibre affect microbiota composition, particularly the abundance of Bacteroides and Bifidobacterium. ${ }^{105}$ In the latter study, body mass index (BMI) did not correlate with microbiota composition. Furthermore, microbial enterotypes were strongly associated with long-term habitual diet, in particular protein and animal fat (Bacteroides) compared with carbohydrate intake (Prevotella). ${ }^{37}$ In line with this, long-term adherence to a Mediterranean diet was associated with specific taxa as well as functionality of the gut microbial profile. ${ }^{106}$ The findings of the latter study suggests that the composition of the gut microbiome is a modulator of the protective association between the Mediterranean diet and cardiometabolic disease risk. ${ }^{106}$ When comparing habitual high-fat diets to high-carbohydrate diets, microbial diversity seems to be lower with high-fat diets. Additionally, microbial diversity seems lower when comparing high (saturated)-fat diets to high carbohydrate/ fibre diets. ${ }^{107-109}$ This diet-induced dysbiosis has been postulated as trigger of metabolic impairments in obesity. ${ }^{7}$ Importantly, evidence linking a high-fat, Western-type diet to the gut microbiota composition and diversity is mostly derived from observational rather than dietary intervention studies. ${ }^{110}$

Although primarily demonstrated in animal models, a limited number of human studies suggest that dietary interventioninduced alterations of microbial composition and functionality may already occur within weeks or even days after changes in dietary intake. ${ }^{111}$ In humans, modest microbial changes were found after a rigorous switch to a solely plant-based or animalbased diet. ${ }^{103}$ These rather extreme forms of dietary interventions provide insights into the mechanisms underlying diet-gut microbiome interactions and show that dietary intervention-induced microbial changes may occur very rapidly. ${ }^{59}$ In line with this, a small controlled-feeding study showed changes in microbiome composition within 24 hours of initiating a high-fat/low-fibre or low-fat/high-fibre diet, although enterotype identity remained stable throughout this 10 -day study. ${ }^{37}$ These findings indicate that there is a tendency for microbial resilience in adults, which might be associated with long-term habitual dietary intake. The concept of bacterial enterotypes, however, has been questioned by several other studies due to the lack of understanding enterotype dynamics and resilience. ${ }^{112}$

Interestingly, a 1-year intervention study comparing the effects of an energy-restricted Mediterranean diet with increased physical activity to an isocaloric Mediterranean diet in overweight/ obese adults showed marked differences in changes of the gut microbiota composition between the groups. Nonetheless, the tendency of microbial shift was in the same direction for both diets. ${ }^{113}$ Besides dietary macronutrient intake, other bioactive compounds may also affect gut microbiome composition and activity. Examples are dietary polyphenols, ${ }^{114}$ micronutrients, vitamins $^{115}$ and probiotics ${ }^{116}$ and may contribute to the gut microbiota-host metabolism cross-talk. In the following paragraphs, we will highlight the effects of dietary macronutrient intake on the human gut microbiota and will link this to host metabolic outcomes. An overview of relevant human studies relating dietary macronutrient or dietary patterns to the gut microbiome is provided in table 1 .

\section{Dietary fibres and metabolic health}

Digestion of carbohydrates is a complex process involving specific enzymes for the type of carbohydrate ingested. The majority of digestible dietary carbohydrates are digested and absorbed in the small intestine, whereas certain, for a large part indigestible carbohydrates, including resistant starch and dietary fibres, are prone to fermentation by gut microbes with the highest content in the colon. Dietary fibres have been shown to have significant effects on the composition and functionality of the gut microbiota associated with health benefits. ${ }^{117-119}$ These dietary fibres may vary in their structural, physical and chemical properties such as water solubility, viscosity, binding and bulking ability, and fermentability. ${ }^{117}$ Highly fermentable fibres, such as $\beta$-glucan, inulin and galacto-oligosaccharides, are well defined in terms of effects on microbiota composition and production of gut metabolites, ${ }^{25}$ while insoluble fibres, although partially fermented, are mostly known for their beneficial effects on stool consistency and colonic transit time. ${ }^{120-122}$

The intake of high-fibre diets beneficially impacts the host's health, among others by affecting glucose and lipid metabolism. Important mechanisms include the regulation of nutrient absorption $^{123}$ or the production of SCFAs, ${ }^{65}$ but data on the health effects of dietary fibres are controversial. There are several explanations for the inconsistent findings in dietary fibre studies. ${ }^{25} 65$ First, in the majority of human studies, only one specific fermentable fibre was supplemented, thereby stimulating only one or a few individual (potentially beneficial) bacteria genera. $^{22} 65$ The consequence of the latter can be a decreased abundance of other essential bacteria, which may result in an imbalance of the microbial ecosystem. Therefore, combining different fibres that stimulate a multitude of different bacterial genera may be important to maintain microbial richness with more pronounced (additive or synergetic) effects on immune status and metabolic health. ${ }^{25}$ Interestingly, a mechanistical study indicated that the site of fibre-derived SCFA production in the colon may be a determinant of metabolic health. The latter study showed that acute distal colonic acetate administration 


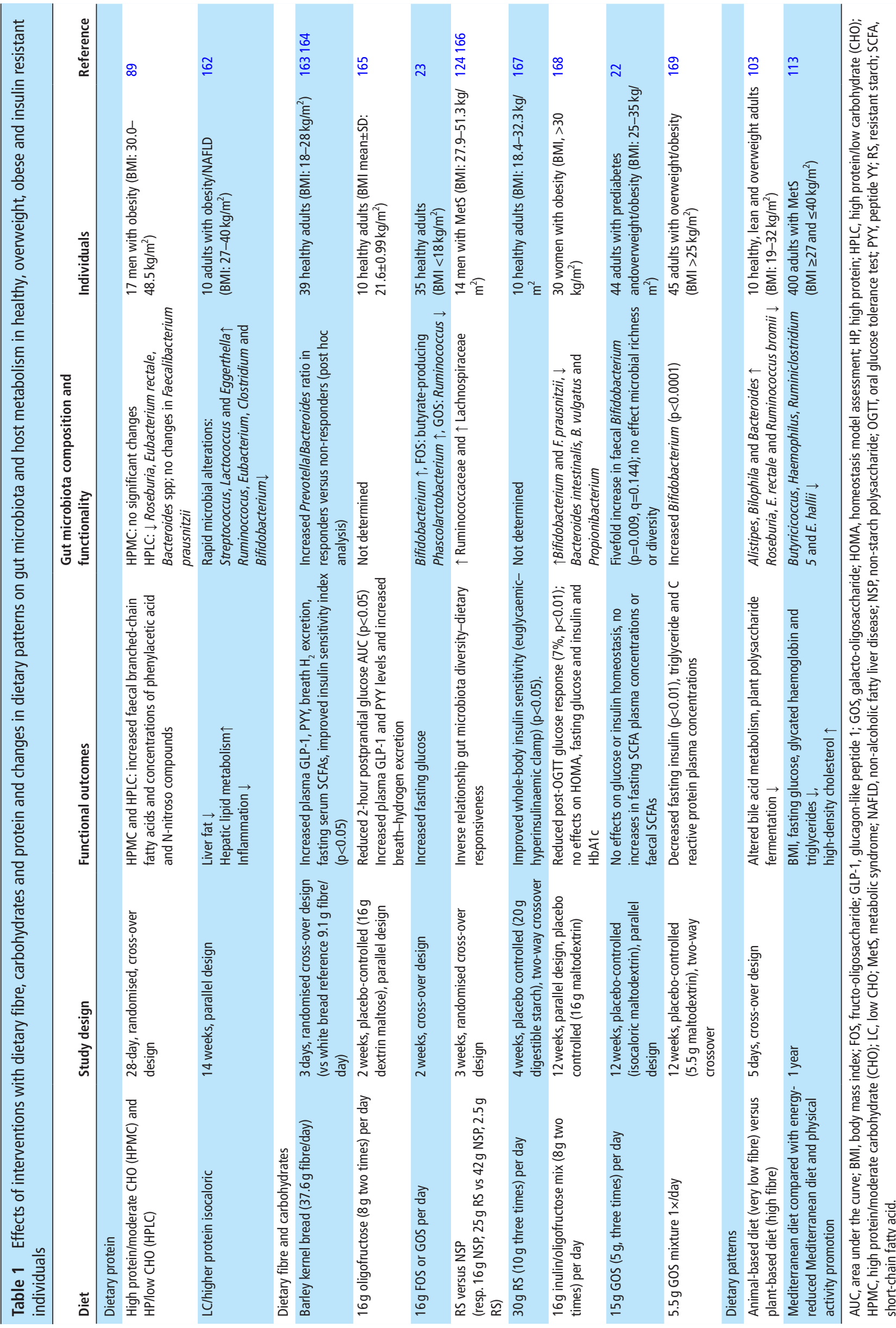


increased circulating acetate concentrations and increased fat oxidation, and satiety-stimulating hormone PYY, and reduced plasma tumour necrosis factor- $\alpha$ in men with overweight. ${ }^{83}$ In contrast to distal infusion, acetate administration in the proximal colon did not affect metabolic profile. ${ }^{83}$ Thus, targeting dietary fibre availability and SCFA formation by microbial species in the distal colon, by combining different dietary fibres and/or more complex dietary fibres, may be a promising strategy to improve immune and metabolic health.

Second, most dietary fibre intervention studies, so far, did not take baseline microbiome or metabolic phenotype into account. Characteristics of the gut microbiome at baseline may strongly interact with dietary intervention outcome. For example, it has been shown that the response of the gut microbiota to dietary fibres (resistant starch vs non-starch polysaccharides) can be predicted from the baseline microbial diversity in men with obesity. ${ }^{124}$ In the latter study, high microbial diversity was associated with lower dietary responsiveness of the microbiota, ${ }^{124}$ which may support the hypothesis that a higher diversity in gut microbes is associated with stability of the microbial ecosystem. In line with this, a high microbial gene count at baseline was related to a less pronounced response to weight loss diets as compared with individuals with a low gene count. In the low gene count group, there was an improvement in gene richness and clinical parameters, although changes in inflammatory markers were less pronounced in individuals with low gene richness. ${ }^{48}$ The basis of the non-response in the high-gene count group is not clear, as this was not associated with habitual dietary intake. Additionally, another study in individuals with obesity showed that not the baseline microbial diversity but rather the baseline abundance of Firmicutes predicted the dietary response of an individual's microbiota. ${ }^{125}$ Together, these findings indicate that microbial diversity is not always a predictor of dietary responsiveness and imply that further research is needed to better understand the complex diet-microbiome-host metabolism interactions.

The efficacy of dietary fibre interventions may also depend on habitual diet and fibre intake. Healthy individuals with high habitual dietary fibre intake had greater changes in gut microbiota composition as a response to an inulin-type fructan prebiotic compared with people with low habitual fibre intake whose gut microbiota seemed to be more resilient to change. ${ }^{126} \mathrm{~A}$ study conducted in patients with T2DM revealed that a select group of SCFA-producing microbial strains was promoted by dietary fibres and that many other microbes, including those in proteolytic fermentation, were either diminished or unchanged, showing an overall decreased microbial gene richness. ${ }^{24}$ Faecal SCFAs increased, in particular butyrate, which was accompanied by improvements in glucose homeostasis. Thus, greater microbial gene richness might not be beneficial per se, as shown in several human fibre dietary intervention studies, ${ }^{24}$ but physiological outcomes may rather be dependent on the functionality of the microbial network.

In a study investigating the effects of a 6-week whole-grain diet on bodyweight changes, a high baseline abundance of bacteria of the Prevotella genus correlated with higher weight loss in overweight, healthy adults. ${ }^{54}$ These findings indicate a predictive capacity as effect modifier of the gut microbiota in response to specific dietary interventions. Additionally, it was found that overweight, prediabetic individuals had a reduced response with respect to changes in microbial diversity and postprandial insulin sensitivity to short-term administration of longchain inulin combined with resistant starch as compared with lean individuals. ${ }^{86}$ In line with this, recent studies have shown that baseline gut microbial signatures may predict the change in
BMI following 3 months' long-chain inulin supplementation, ${ }^{127}$ an effect that was replicated in hum-ob mice colonised with the faecal microbiota from different individuals with obesity at baseline.

Overall, the effectiveness of prebiotic dietary fibres on metabolic health outcomes may depend on several parameters, including initial microbial composition as well as the site of microbial fermentation.

Dietary protein, microbiota composition and metabolic health Ingested dietary proteins are first digested in the small intestine by pancreatic enzymes and peptidases from enterocytes. A significant amount of oligopeptides and amino acids is then transported to the portal bloodstream via enterocyte transporters where they are used as amino acid precursors for protein synthesis or metabolised for fuels or precursors necessary for intestinal mucosal metabolites. ${ }^{88} 128129$ Since fermentable carbohydrates are preferentially used over proteins by most bacteria in the distal small intestine and proximal colon, most fermentation of amino acids as an energy source occurs in the distal colon, where carbohydrates are depleted. ${ }^{130}$ The percentage of ingested protein reaching the large intestine is likely also dependent on protein quality, with estimated numbers around $10 \% .{ }^{131}$ Protein originating from plants have a lower digestibility due to the indigestible cell wall of the plant, ${ }^{132}$ while protein derived from animals can be more easily digested in the large intestine, indicating potential difference in functional outcomes. Metabolites resulting from proteolysis (figure 1) can be either used by colonocytes or excreted in faeces. ${ }^{128}$ Most proteolytic products are considered to have detrimental effects on gut and host metabolic health. ${ }^{89}$ Furthermore, the balance between proteolytic and saccharolytic fermentation may determine the non-response to lifestyle interventions ${ }^{133}$ and should therefore be considered in future research. ${ }^{134}$

Metabolic effects of protein intake have been well studied. Casein, a relatively slowly digestible protein derived from animal products, was the most efficient protein source preventing weight gain and accretion of adipose mass in mice on a high-fat/ high-protein diet (HPD). ${ }^{135}$ Epidemiological studies also indicate that intake of dairy and vegetarian protein sources is associated with protection against obesity, whereas a high intake of meat (in particular red meat) predicts higher weight gain. ${ }^{136-138}$ Although investigated less extensively, protein intake has been shown to affect microbiota composition and functionality. ${ }^{139}$ Effects are determined by the amino acid composition and digestibility of proteins, which are influenced by the source and the amount of intake. ${ }^{140}$ High protein diets have been linked to reductions in C. coccoides, C. leptum and F. prausnitzii in rats, ${ }^{141}$ while reductions in Roseburia spp, E. rectale, C. aerofaciens, Bacteroides spp and Oscillibacter relatives have been observed in men with overweight or obesity. ${ }^{89} 142$ Of note, comparing HPDs to moderateprotein or low-protein diets in an isocaloric manner induces differences in either carbohydrate or fat content between diets. As for all isocaloric dietary macronutrient exchange studies, it is therefore difficult to determine the predominant dietary factor responsible for compositional shifts in the gut microbiota, which could be attributed to either an increase in one (macro) nutrient or a decrease in the other.

Although proteolytic metabolites are predominantly associated with adverse health effects, current evidence also suggests that proteolytic metabolites may have beneficial effects on metabolic health. For instance, several amino acid-derived compounds that can be produced only by gut bacteria (indole) or by mammalian 
host (tyramine, tryptamine and SCFAs) (in)directly impact satiety and gut motility in mammals via effects on GLP-1 and serotonin secretion from enteroendocrine cells. ${ }^{143}$ However, the physiological effect on the host intestinal and peripheral tissues of the majority of these compounds is still not well understood. In addition, the distinction between human and bacterial origins of many of these compounds has not been well established yet, and further in vivo studies are necessary to validate such effects.

\section{Dietary fats and polyphenols and interaction with the gut microbiome}

Dietary fats have been studied extensively in relation to dietrelated metabolic diseases such as obesity, but the effects on the gut microbiota are less defined in humans, and studies often yield opposite findings. ${ }^{144}$ FAs differing in type (saturated vs monosaturated and polyunsaturated FAs), carbon chain length and the degree of saturation may have a distinct effect on the gut microbiota composition. ${ }^{109}$ Cross-sectional studies indicate that consumption of a diet rich in animal protein and fat was associated with the Bacteroides enterotype, while higherfibre, fruit and vegetable consumption was associated with the Prevotella enterotype in healthy adults. ${ }^{145}$ Furthermore, a high intake of mainly saturated fatty acids (SFAs) has been associated with a decrease in gut microbial richness and diversity in both adults and infants. ${ }^{146147}$ In people with overweight and obesity, SFAs were negatively associated with the genus Intestinimonas, whereas SFAs were positively associated with Roseburia, which was also highly abundant in individuals with normal body weight. ${ }^{50}$ In this study, habitual SFA intake exhibited opposite association profiles with butyrate-producing bacteria, depending on the BMI. Overall, it should be noted that dietary fat-microbiome-host physiology interactions are studied less extensively compared with dietary fibre, and that the mechanistic knowledge is largely based on animal studies. Interpretation of these findings in light of human physiology is difficult and should be investigated further.

Second, out of a wide array of dietary components, dietary polyphenols and their effect on the gut microbiome and host metabolism have been studied extensively in recent years. Polyphenols, present as phenolic compounds in mainly fruits and vegetables, are known for their beneficial effects as antioxidant, anti-inflammatory, cardioprotective, cancer preventive and neuroprotective agents. ${ }^{148}$ A 2-month epigallocatechin-3-gallate supplementation, naturally present in tea, had both an effect on bile acid metabolism and Verrucomicrobiaceae abundance in mice with obesity, under which it promoted the increase of $A$. muciniphila abundance. ${ }^{149}$ The latter has been associated with beneficial metabolic effects in other studies. ${ }^{52150151}$ Additionally, although also in an animal model, 8-week polyphenol supplementation protected from diet-induced obesity, IR and intestinal inflammation, in association with increased abundance of Akkermansia spp. ${ }^{114}$ In healthy, overweight or obese individuals, 12-week combined resveratrol and epigallocatechin-3-gallate supplementation improved both metabolic parameters and decreased Bacteroidetes in men but not in women. ${ }^{152}$ Overall, adding dietary polyphenols to a diet seems to promote both gut and metabolic health, although mechanistical insights in human studies are still required.

\section{TOWARDS PRECISION NUTRITION: CONCLUSIONS AND FUTURE PERSPECTIVES}

Microbiome-based precision nutrition to predict metabolic health parameters like glycaemic response and variability, or used to counteract metabolic disarrangements has currently received major scientific attention. A landmark study within this field showed that, despite high interpersonal variability in postprandial glucose response, personalised diets (based on habitual diet, physical activity and gut microbiota) created with the help of a machine-learning algorithm may successfully lower blood glucose responses. ${ }^{39}$ The postprandial predictive capacity of the gut microbiome to meal challenges was again highlighted in another study, showing that an individual's microbiome had a greater influence (explaining $7.1 \%$ of variance) than meal macronutrients (explaining 3.6\% of variance) for predicting postprandial lipaemia, but not for postprandial glycaemia (explaining $6.0 \%$ and $15.4 \%$ of variance, respectively). ${ }^{38}$ In line with this, significant interpersonal differences were found in glycaemic response to different types of bread, and this glycaemic response could be predicted by baseline microbiome features. ${ }^{153}$ Of note, these studies mainly based their findings on responses to acute meal challenges and short-term interventions rather than on longer-term intervention responses. Conclusive results for the latter is still scarce, but the predictive capacity of the gut microbiome is becoming more evident, especially in studies examining the effects of fibre and faecal microbiota transplantation. ${ }^{13} 1440$

As discussed previously, there may be putative resistance of the microbiome-host metabolism axis to dietary intervention in individuals with IR, suggesting that interventions may require longer periods of time or that higher intake of functional dietary components such as dietary fibre is needed to induce beneficial effects. In this respect, the baseline microbial profile is an interesting biomarker for responsiveness to dietary interventions (eg, dietary fibre or complex proteins). Specific functional microbial groups are characterised by distinct digestive capacities for dietary components, resulting in a differential production of microbial metabolites such as SCFAs, subsequently impacting regulation of the host's metabolism. ${ }^{154}$ Several studies identified a predictive capacity for a Prevotella enterotype to optimise weight loss in response to a high-fibre diet, while this was not found in individuals with a Bacteroidetes enterotype. ${ }^{4054155}$ The effectiveness of administering optimal fibre types in individuals stratified for microbial composition and functionality, degree of diversity or abundance of specific microbial strains has not been examined yet; therefore, prospective evidence is required in future dietary intervention studies. Overall, when evaluating dietary patterns and diets varying in macronutrient composition, it is important to take both quantity as well as quality of dietary components, micronutrient as well as bioactive components like polyphenols into account-within a whole-diet approach-due to differential interaction with the host's microbial and metabolic phenotype (figure 2).

The responsiveness to dietary interventions does not solely depend on characteristics of the gut microbiota but is rather dependent on a complex, multifactorial interaction between diet, lifestyle and environmental factors and clinical characteristics such as the metabolic phenotype (figure 2). ${ }^{16156157} \mathrm{It}$ was recently shown that men and women differ in microbiota composition and that this composition is associated with insulin sensitivity in men but not in women. ${ }^{158}$ Interestingly, another study also demonstrated that 12-week combined polyphenol supplementation (using epigallocatechin-gallate (EGCG) and resveratrol) affected the gut microbiota composition in men but not in women. ${ }^{152}$ Both studies indicate a sex-specific microbial response, which should be considered when evaluating intervention responses.

In a study investigating metabolic responsiveness to specific diets in different metabolic phenotypes, the presence of either 


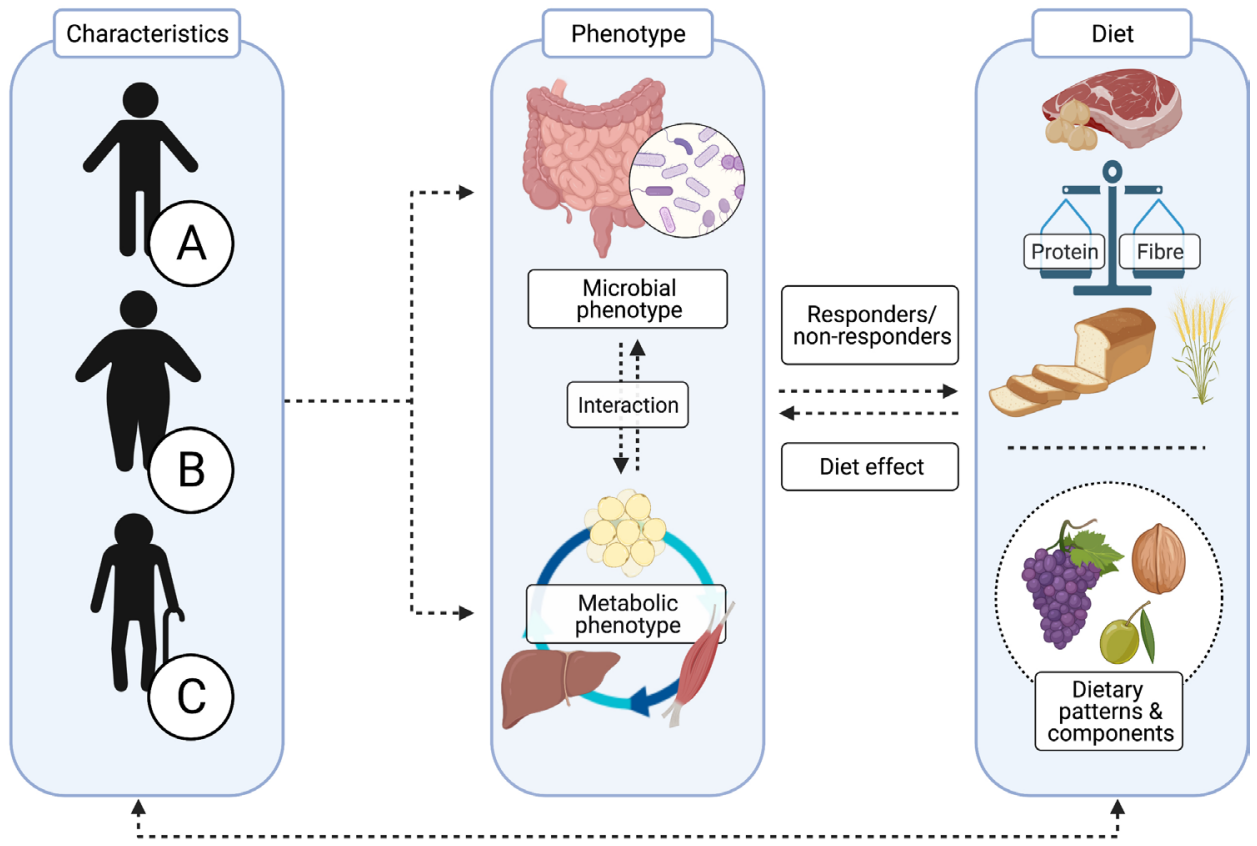

Figure 2 Applying optimal strategies in precision nutrition requires detailed characterisation of the individual. The composition and functionality of the gut microbiota are influenced by many factors, including genetics, age, sex and environmental factors such as the mode of delivery, drug use, disease, geography, physical exercise and diet. ${ }^{19}$ The multidirectional interaction between the gut microbiota, host metabolism and diet is therefore complex and highly individualised. When developing more targeted dietary strategies for individuals A, B and C, the microbial and metabolic phenotypes (eg, tissue-specific insulin resistance), as well as characteristics of dietary components (including the balance between protein and carbohydrate (CHO)/fibre intake) should be well considered.

more pronounced muscle IR on more pronounced hepatic IR at baseline was a determinant of the change in disposition index (a composite marker of insulin sensitivity and insulin secretion) after 2 years of either a Mediterranean or a low-fat diet. ${ }^{159}$ Individuals with muscle IR responded better to the Mediterranean diet, while individuals with hepatic IR were more responsive to the low-fat diet. ${ }^{159}$ This study illustrates the importance of considering individual metabolic characteristics when developing precision nutrition strategies. To which extent the gut microbiome is involved in these types of dietary interventions remains to be investigated. Interestingly, the soluble fibre inulin has been shown to reduce IR in people with impaired fasting glucose but not in those with impaired glucose tolerance. The latter findings may suggest tissue specificity in the fibre-gut microbiota-host metabolism crosstalk, in view of the findings that impaired fasting glucose is strongly related to hepatic IR. ${ }^{160}$ Of note, targeting metabolically impaired individuals indeed requires both detailed characterisation of an individual's microbial and metabolic phenotyping, as well as lifestyle factors. A previous study has shown that associations between microbial taxa and tissue-specific insulin sensitivity might be cohortspecific, suggesting alternative states of dysbiosis, depending on cohort characteristics. This further highlights the complexity of interpretation and generalisation of findings in the gut microbiome field. ${ }^{161}$

In order to translate precision-based strategies into healthcare practice or into guidelines, we need a thorough understanding of why people respond differentially to diet, whether differential responses and related phenotypes are maintained in the long term and to what extent developed algorithms are reproducible. In our view, detailed microbial and metabolic phenotyping by state-of-the art methodologies in dietary intervention studies is crucial. Obviously, in view of the complexity, we need besides that detailed information about lifestyle and environmental factors, including mode of birth, medical history, use of medication, in particular antibiotics, physical activity, psychological stress and sleep quality. This also implies that we need advanced statistical and modelling methodologies to tease out the importance of different factors. Importantly, however, we certainly must go beyond a black-box machine-learning prediction of dietary intervention responses.

Overall, we conclude that current evidence for developing optimal dietary interventions targeting bodyweight control and IR via the gut microbiota is still in its infancy and does not capture the complexity of the integration of a whole-diet approach, the microbial and the host's metabolic phenotype (figure 2). Implementation of targeted, precision nutrition intervention strategies or dietary guidelines for individuals or subgroups in public health is still more remote and will require insight in the mechanisms involved in (non-)response to dietary intervention, implying that we need to go beyond prediction models. Detailed individual phenotyping and gaining insights into the balance between carbohydrate and protein fermentation by the gut microbiota as well as the site of fermentation in the colon are thereby key.

\section{DATA SOURCES}

A semisystematic search in PubMed was used to select relevant literature. Literature was managed using EndNote V.X8. The search strategy consisted of one or a combination of the following search terms using the "AND" and "OR" operators:

"Insulin resistance"[MeSH], "obesity" $[\mathrm{MeSH}]$, "gut microbiota" $[\mathrm{MeSH}]$, "type 2 diabetes" $[\mathrm{MeSH}]$, "short-chain fatty acids" [TIAB], “lps"[TIAB], "glucose metabolism”[TIAB], "”[TIAB], "impaired fasting glucose"[TIAB], "impaired glucose tolerance"[TIAB], “energy metabolism”[Mesh], “gut-brain axis"[TIAB], "PYY”[TIAB], "GLP-1"[TIAB], "peripheral insulin 
resistance"[TIAB], “insulin"[TIAB], “gut metabolites”[TIAB], "enterotypes"[TIAB], "gut microbiota composition"[TIAB], "dietary proteins”[MeSH], “dietary carbohydrates"[MeSH], "dietary fiber" $[\mathrm{MeSH}]$, “dietary fat" $[\mathrm{MeSH}]$, “diet”[MeSH], "dietary patterns" $[\mathrm{MeSH}]$. From the identified articles, the titles and abstracts were assessed, and if considered relevant for the present review, the full text of the article was examined in detail. In addition, additional records were identified through searching reference lists. Only articles written in English were included.

Correction notice This article has been corrected since it published Online First. The author affiliations have been updated.

Acknowledgements The authors acknowledge BioRender.com as the tool for creating the figures in this article.

Contributors KMJ wrote the manuscript; EEC, GHG and EEB conceptualised, reviewed and edited the manuscript. All authors read and approved the final version of the manuscript.

Funding The authors performed their work within a project organised by and executed under the auspices of TiFN, a public-private partnership on precompetitive research in food and nutrition (project code: 16NH04). Funding for this research was obtained from the Netherlands Organization for Scientific Research, DSM, FrieslandCampina, Danone Nutricia Research, and the Top Sector Agri \& Food the Netherlands.

\section{Competing interests None declared.}

Patient and public involvement Patients and/or the public were not involved in the design, conduct, reporting or dissemination plans of this research.

Patient consent for publication Not applicable.

Ethics approval This study does not involve human participants.

Provenance and peer review Not commissioned; externally peer reviewed.

Open access This is an open access article distributed in accordance with the Creative Commons Attribution Non Commercial (CC BY-NC 4.0) license, which permits others to distribute, remix, adapt, build upon this work non-commercially, and license their derivative works on different terms, provided the original work is properly cited, appropriate credit is given, any changes made indicated, and the use is non-commercial. See: http://creativecommons.org/licenses/by-nc/4.0/.

\section{ORCID iDs}

Kelly M Jardon http://orcid.org/0000-0001-8859-375X

Emanuel E Canfora http://orcid.org/0000-0003-0267-1097

Giis H Goossens http://orcid.org/0000-0002-2092-3019

Ellen E Blaak http://orcid.org/0000-0002-2496-3464

\section{REFERENCES}

1 Zheng Y, Ley SH, Hu FB. Global aetiology and epidemiology of type 2 diabetes mellitus and its complications. Nat Rev Endocrinol 2018;14:88-98.

2 Tremmel M, Gerdtham U-G, Nilsson PM, et al. Economic burden of obesity: a systematic literature review. Int J Environ Res Public Health 2017;14. doi:10.3390/ ijerph14040435. [Epub ahead of print: 1904 2017].

3 Anderson JW, Konz EC, Frederich RC, et al. Long-Term weight-loss maintenance: a meta-analysis of US studies. Am J Clin Nutr 2001;74:579-84

4 Le Chatelier E, Nielsen T, Qin J, et al. Richness of human gut microbiome correlates with metabolic markers. Nature 2013:500:541-6.

5 Turnbaugh PJ, Hamady M, Yatsunenko T, et al. A core gut microbiome in obese and lean twins. Nature 2009:457:480-4.

6 Turnbaugh PJ, Bäckhed F, Fulton L, et al. Diet-Induced obesity is linked to marked but reversible alterations in the mouse distal gut microbiome. Cell Host Microbe 2008:3:213-23.

7 Ley RE, Turnbaugh PJ, Klein S, et al. Microbial ecology: human gut microbes associated with obesity. Nature 2006;444:1022-3.

8 Schroeder BO, Bäckhed F. Signals from the gut microbiota to distant organs in physiology and disease. Nat Med 2016;22:1079-89.

9 Schwiertz A, Taras D, Schäfer K, et al. Microbiota and SCFA in lean and overweight healthy subjects. Obesity 2010;18:190-5.

10 Bouter KE, van Raalte DH, Groen AK, et al. Role of the gut microbiome in the pathogenesis of obesity and obesity-related metabolic dysfunction. Gastroenterology 2017;152:1671-8.

11 Nguyen TLA, Vieira-Silva S, Liston A, et al. How informative is the mouse for human gut microbiota research? Dis Model Mech 2015;8:1-16.

12 Savage DC. Microbial biota of the human intestine: a tribute to some pioneering scientists. Curr Issues Intest Microbiol 2001;2:1-15.
13 Vrieze A, Van Nood E, Holleman F, et al. Transfer of intestinal microbiota from lean donors increases insulin sensitivity in individuals with metabolic syndrome. Gastroenterology 2012;143:913-6.

14 Kootte RS, Levin E, Salojärvi J, et al. Improvement of insulin sensitivity after lean donor feces in metabolic syndrome is driven by baseline intestinal microbiota composition. Cell Metab 2017:26:611-9.

15 Reijnders D, Goossens GH, Hermes GDA, et al. Effects of gut microbiota manipulation by antibiotics on host metabolism in obese humans: a randomized double-blind placebo-controlled trial. Cell Metab 2016;24:63-74.

16 Rothschild D, Weissbrod O, Barkan E, et al. Environment dominates over host genetics in shaping human gut microbiota. Nature 2018;555:210-5.

17 Conlon MA, Bird AR. The impact of diet and lifestyle on gut microbiota and human health. Nutrients 2014;7:17-44.

18 Dominianni C, Sinha R, Goedert JJ, et al. Sex, body mass index, and dietary fiber intake influence the human gut microbiome. PLoS One 2015;10:e0124599.

19 Quigley EMM. Gut microbiome as a clinical tool in gastrointestinal disease management: are we there yet? Nat Rev Gastroenterol Hepatol 2017:14:315-20.

20 Portune KJ, Benítez-Páez A, Del Pulgar EMG, et al. Gut microbiota, diet, and obesityrelated disorders-The good, the bad, and the future challenges. Mol Nutr Food Res 2017;61. doi:10.1002/mnfr.201600252. [Epub ahead of print: 2009 2016]

21 Holscher HD. Dietary fiber and prebiotics and the gastrointestinal microbiota. Gut Microbes 2017:8:172-84.

22 Canfora EE, van der Beek CM, Hermes GDA, et al. Supplementation of Diet With Galacto-oligosaccharides Increases Bifidobacteria, but Not Insulin Sensitivity, in Obese Prediabetic Individuals. Gastroenterology 2017;153:87-97.

23 Liu F, Li P, Chen M, et al. Fructooligosaccharide (Fos) and Galactooligosaccharide (GOS) increase Bifidobacterium but reduce butyrate producing bacteria with adverse glycemic metabolism in healthy young population. Sci Rep 2017:7:11789.

24 Zhao L, Zhang F, Ding X. Gut bacteria selectively promoted by dietary fibers alleviate type 2 diabetes. Science 2018;359:1151-6.

25 Canfora EE, Meex RCR, Venema K, et al. Gut microbial metabolites in obesity, NAFLD and T2DM. Nat Rev Endocrinol 2019;15:261-73.

26 Agus A, Clément K, Sokol H. Gut microbiota-derived metabolites as central regulators in metabolic disorders. Gut 2021;70:1174-82

27 Khosravi A, Mazmanian SK. Disruption of the gut microbiome as a risk factor for microbial infections. Curr Opin Microbiol 2013;16:221-7.

28 Vrieze A, Out C, Fuentes S, et al. Impact of oral vancomycin on gut microbiota, bile acid metabolism, and insulin sensitivity. J Hepatol 2014;60:824-31.

29 Arumugam M, Raes J, Pelletier $\mathrm{E}$, et al. Enterotypes of the human gut microbiome. Nature 2011;473:174-80.

30 Goodrich JK, Waters JL, Poole AC, et al. Human genetics shape the gut microbiome. Cell 2014;159:789-99.

31 Markle JGM, Frank DN, Mortin-Toth S, et al. Sex differences in the gut microbiome drive hormone-dependent regulation of autoimmunity. Science 2013;339:1084-8

32 Org E, Mehrabian M, Parks BW, et al. Sex differences and hormonal effects on gut microbiota composition in mice. Gut Microbes 2016:7:313-22.

33 Yatsunenko T, Rey FE, Manary MJ, et al. Human gut microbiome viewed across age and geography. Nature 2012:486:222-7.

34 Odamaki T, Kato K, Sugahara H, et al. Age-Related changes in gut microbiota composition from newborn to centenarian: a cross-sectional study. BMC Microbiol 2016;16:90.

35 Gupta VK, Paul S, Dutta C, Geography DC. Geography, ethnicity or SubsistenceSpecific variations in human microbiome composition and diversity. Front Microbiol 2017:8:1162.

36 Imhann F, Bonder MJ, Vich Vila A, et al. Proton pump inhibitors affect the gut microbiome. Gut 2016;65:740-8.

37 Wu GD, Chen J, Hoffmann C, et al. Linking long-term dietary patterns with gut microbial enterotypes. Science 2011;334:105-8.

38 Berry SE, Valdes AM, Drew DA, et al. Human postprandial responses to food and potential for precision nutrition. Nat Med 2020;26:964-73.

39 Zeevi D, Korem T, Zmora N, et al. Personalized nutrition by prediction of glycemic responses. Cell 2015;163:1079-94.

40 Hjorth MF, Blædel T, Bendtsen LQ, et al. Prevotella-to-Bacteroides ratio predicts body weight and fat loss success on 24-week diets varying in macronutrient composition and dietary fiber: results from a post-hoc analysis. Int I Obes 2019;43:149-57.

41 Turnbaugh PJ, Ley RE, Mahowald MA, et al. An obesity-associated gut microbiome with increased capacity for energy harvest. Nature 2006;444:1027-31.

42 Ley RE, Bäckhed F, Turnbaugh P, et al. Obesity alters gut microbial ecology. Proc Natl Acad Sci U S A 2005;102:11070-5.

43 Tims S, Derom C, Jonkers DM, et al. Microbiota conservation and BMI signatures in adult monozygotic twins. Isme J 2013;7:707-17.

44 Duncan SH, Lobley GE, Holtrop G, et al. Human colonic microbiota associated with diet, obesity and weight loss. Int J Obes 2008;32:1720-4.

45 Aron-Wisnewsky J, Prifti E, Belda E, et al. Major microbiota dysbiosis in severe obesity: fate after bariatric surgery. Gut 2019;68:70-82.

46 Sanna S, van Zuydam NR, Mahajan A, et al. Causal relationships among the gut microbiome, short-chain fatty acids and metabolic diseases. Nat Genet 2019;51:600-5 
47 Larsen N, Vogensen FK, van den Berg FWJ, et al. Gut microbiota in human adults with type 2 diabetes differs from non-diabetic adults. PLoS One 2010;5:e9085.

48 Cotillard A, Kennedy SP, Kong LC, et al. Dietary intervention impact on gut microbial gene richness. Nature 2013:500:585-8.

49 Kim M-H, Yun KE, Kim J, et al. Gut microbiota and metabolic health among overweight and obese individuals. Sci Rep 2020;10:19417.

50 Companys J, Gosalbes MJ, Pla-Pagà L, et al. Gut microbiota profile and its association with clinical variables and dietary intake in overweight/obese and lean subjects: a cross-sectional study. Nutrients 2021;13:13.

51 Zhou Q, Zhang Y, Wang X, et al. Gut bacteria Akkermansia is associated with reduced risk of obesity: evidence from the American Gut Project. Nutr Metab 2020:17:90.

52 Zhou Q, Pang G, Zhang Z, et al. Association Between Gut Akkermansia and Metabolic Syndrome is Dose-Dependent and Affected by Microbial Interactions: A Cross-Sectional Study. Diabetes Metab Syndr Obes 2021;14:2177-88.

53 Jie Z, Yu X, Liu Y, et al. The baseline gut microbiota directs Dieting-Induced weight loss trajectories. Gastroenterology 2021;160:e16:2029-42.

54 Christensen L, Vuholm S, Roager HM, et al. Prevotella abundance predicts weight loss success in healthy, overweight adults consuming a Whole-Grain diet AD libitum: a post hoc analysis of a 6-Wk randomized controlled trial. J Nutr 2019;149:2174-81.

55 Qin J, Li Y, Cai Z, et al. A metagenome-wide association study of gut microbiota in type 2 diabetes. Nature 2012;490:55-60.

56 Karlsson FH, Tremaroli V, Nookaew I, et al. Gut metagenome in European women with normal, impaired and diabetic glucose control. Nature 2013;498:99-103.

57 Pedersen HK, Gudmundsdottir V, Nielsen HB, et al. Human gut microbes impact host serum metabolome and insulin sensitivity. Nature 2016;535:376-81.

58 Fan Y, Pedersen 0. Gut microbiota in human metabolic health and disease. Nat Rev Microbiol 2021;19:55-71.

59 Klurfeld DM, Davis CD, Karp RW, et al. Considerations for best practices in studies of fiber or other dietary components and the intestinal microbiome. Am J Physiol Endocrinol Metab 2018;315:E1087-97.

60 Wong JMW, de Souza R, Kendall CWC, et al. Colonic health: fermentation and short chain fatty acids. J Clin Gastroenterol 2006;40:235-43.

61 Louis P, Flint HJ. Formation of propionate and butyrate by the human colonic microbiota. Environ Microbiol 2017;19:29-41.

62 Rivière A, Selak M, Lantin D, et al. Bifidobacteria and butyrate-producing colon bacteria: importance and strategies for their stimulation in the human gut. Front Microbiol 2016:7:979

63 Boets E, Gomand SV, Deroover L, et al. Systemic availability and metabolism of colonic-derived short-chain fatty acids in healthy subjects: a stable isotope study. J Physiol 2017;595:541-55.

64 Blaut M. Gut microbiota and energy balance: role in obesity. Proc Nutr Soc 2015;74:227-34

65 Canfora EE, Jocken JW, Blaak EE. Short-Chain fatty acids in control of body weight and insulin sensitivity. Nat Rev Endocrinol 2015;11:577-91.

66 Koh A, De Vadder F, Kovatcheva-Datchary P, et al. From dietary fiber to host physiology: short-chain fatty acids as key bacterial metabolites. Cell 2016;165:1332-45

67 Aberdein N, Schweizer M, Ball D. Sodium acetate decreases phosphorylation of hormone sensitive lipase in isoproterenol-stimulated 3T3-L1 mature adipocytes. Adipocyte 2014;3:121-5.

68 Jocken JWE, González Hernández MA, Hoebers NTH, et al. Short-Chain fatty acids differentially affect intracellular lipolysis in a human white adipocyte model. Front Endocrinol 2017:8:372.

69 Ohira H, Fujioka Y, Katagiri C, et al. Butyrate attenuates inflammation and lipolysis generated by the interaction of adipocytes and macrophages. J Atheroscler Thromb 2013;20:425-42

70 Gao Z, Yin J, Zhang J, et al. Butyrate improves insulin sensitivity and increases energy expenditure in mice. Diabetes 2009;58:1509-17.

71 Yamashita $\mathrm{H}$, Maruta $\mathrm{H}$, Jozuka $\mathrm{M}$, et al. Effects of acetate on lipid metabolism in muscles and adipose tissues of type 2 diabetic Otsuka Long-Evans Tokushima fatty (OLETF) rats. Biosci Biotechnol Biochem 2009:73:570-6.

72 Priyadarshini M, Villa SR, Fuller M, et al. An Acetate-Specific GPCR, FFAR2, regulates insulin secretion. Mol Endocrinol 2015;29:1055-66.

73 McNelis JC, Lee YS, Mayoral R, et al. Gpr43 potentiates $\beta$-cell function in obesity. Diabetes 2015;64:3203-17.

74 den Besten G, Bleeker A, Gerding A, et al. Short-Chain fatty acids protect against high-fat diet-induced obesity via a PPAR $\gamma$-dependent switch from lipogenesis to fat oxidation. Diabetes 2015:64:2398-408.

75 Li X, Chen H, Guan Y, et al. Acetic acid activates the AMP-activated protein kinase signaling pathway to regulate lipid metabolism in bovine hepatocytes. PLoS One 2013;8:e67880.

76 De Vadder F, Kovatcheva-Datchary P, Goncalves D, et al. Microbiota-generated metabolites promote metabolic benefits via gut-brain neural circuits. Cell 2014;156:84-96.

77 Goswami C, Iwasaki Y, Yada T. Short-Chain fatty acids suppress food intake by activating vagal afferent neurons. J Nutr Biochem 2018;57:130-5.
78 Li Z, Yi C-X, Katiraei S, et al. Butyrate reduces appetite and activates brown adipose tissue via the gut-brain neural circuit. Gut 2018:67:1269-79.

79 Liu Z, Dai X, Zhang H, et al. Gut microbiota mediates intermittent-fasting alleviation of diabetes-induced cognitive impairment. Nat Commun 2020;11:855.

80 Petersen KF, Impellizeri A, Cline GW, et al. The effects of increased acetate turnove on glucose-induced insulin secretion in lean and obese humans. J Clin Trans/ SCi 2019:3:18-20.

81 Chambers ES, Viardot A, Psichas A, et al. Effects of targeted delivery of propionate to the human colon on appetite regulation, body weight maintenance and adiposity in overweight adults. Gut 2015;64:1744-54.

82 Canfora $\mathrm{EE}$, van der Beek CM, Jocken JWE, et al. Colonic infusions of short-chain fatty acid mixtures promote energy metabolism in overweight/obese men: a randomized crossover trial. Sci Rep 2017;7:2360.

83 van der Beek CM, Canfora EE, Lenaerts K, et al. Distal, not proximal, colonic acetate infusions promote fat oxidation and improve metabolic markers in overweight/obese men. Clin Sci 2016;130:2073-82.

84 Sukkar $\mathrm{AH}$, Lett $\mathrm{AM}$, Frost $\mathrm{G}$, et al. Regulation of energy expenditure and substrate oxidation by short-chain fatty acids. J Endocrinol 2019;242:R1-8.

85 Chambers ES, Byrne CS, Aspey K, et al. Acute oral sodium propionate supplementation raises resting energy expenditure and lipid oxidation in fasted humans. Diabetes Obes Metab 2018;20:1034-9.

86 Blaak EE. Current metabolic perspective on malnutrition in obesity: towards more subgroup-based nutritional approaches? Proc Nutr Soc 2020;79:1-7.

87 Blaak EE, Canfora EE, Theis S, et al. Short chain fatty acids in human gut and metabolic health. Benef Microbes 2020;11:411-55.

88 Windey K, De Preter V, Verbeke K. Relevance of protein fermentation to gut health. Mol Nutr Food Res 2012;56:184-96.

89 Russell WR, Gratz SW, Duncan SH, et al. High-Protein, reduced-carbohydrate weightloss diets promote metabolite profiles likely to be detrimental to colonic health. Am J Clin Nutr 2011:93:1062-72.

90 Dodd D, Spitzer MH, Van Treuren W, et al. A gut bacterial pathway metabolizes aromatic amino acids into nine circulating metabolites. Nature 2017;551:648-52.

91 Okamoto M, Yamaoka M, Takei M, et al. Endogenous hydrogen sulfide protects pancreatic beta-cells from a high-fat diet-induced glucotoxicity and prevents the development of type 2 diabetes. Biochem Biophys Res Commun 2013:442:227-33.

92 Beaumont M, Neyrinck AM, Olivares M, et al. The gut microbiota metabolite indole alleviates liver inflammation in mice. Faseb J 2018:6681-93.

93 Jha R, Leterme P. Feed ingredients differing in fermentable fibre and indigestible protein content affect fermentation metabolites and faecal nitrogen excretion in growing pigs. Animal 2012;6:603-11

94 Randrianarisoa $\mathrm{E}$, Lehn-Stefan A, Wang $\mathrm{X}$, et al. Relationship of serum trimethylamine $\mathrm{N}$-oxide (TMAO) levels with early atherosclerosis in humans. Sci Rep 2016:6:26745

95 Koeth RA, Wang Z, Levison BS, et al. Intestinal microbiota metabolism of L-carnitine, a nutrient in red meat, promotes atherosclerosis. Nat Med 2013;19:576.

96 Chen Y-ming, Liu Y, Zhou R-fen, Y-m C, R- $f$ Z, et al. Associations of gut-floradependent metabolite trimethylamine- $\mathrm{N}$-oxide, betaine and choline with nonalcoholic fatty liver disease in adults. Sci Rep 2016:6:19076.

97 Gao X, Liu X, Xu J, et al. Dietary trimethylamine N-oxide exacerbates impaired glucose tolerance in mice fed a high fat diet. J Biosci Bioeng 2014:118:476-81.

98 Heianza Y, Sun D, Li X, et al. Gut microbiota metabolites, amino acid metabolites and improvements in insulin sensitivity and glucose metabolism: the pounds lost trial. Gut 2019:68:263-70.

99 Shan Z, Sun T, Huang H, et al. Association between microbiota-dependent metabolite trimethylamine-N-oxide and type 2 diabetes. Am J Clin Nutr 2017;106:888-94.

100 Li Q, Wu T, Liu R. Soluble dietary fiber reduces trimethylamine metabolism via gut microbiota and co-regulates host AMPK pathways. Molecular nutrition \& food research 2017;61:1700473.

101 Dong TS, Gupta A. Influence of early life, diet, and the environment on the microbiome. Clin Gastroenterol Hepatol 2019;17:231-42.

102 Falony G, Joossens M, Vieira-Silva S, et al. Population-Level analysis of gut microbiome variation. Science 2016;352:560-4

103 David LA, Maurice CF, Carmody RN, et al. Diet rapidly and reproducibly alters the human gut microbiome. Nature 2014;505:559-63.

104 Zhernakova A, Kurilshikov A, Bonder MJ, et al. Population-Based metagenomics analysis reveals markers for gut microbiome composition and diversity. Science 2016;352:565-9

105 Simões CD, Maukonen J, Kaprio J, et al. Habitual dietary intake is associated with stool microbiota composition in monozygotic twins. J Nutr 2013;143:417-23.

106 Wang DD, Nguyen LH, Li Y, et al. The gut microbiome modulates the protective association between a Mediterranean diet and cardiometabolic disease risk. Nat Med 2021:27:333-43.

107 De Filippo C, Cavalieri D, Di Paola M, et al. Impact of diet in shaping gut microbiota revealed by a comparative study in children from Europe and rural Africa. Proc Nat Acad Sci U S A 2010:107:14691-6.

108 Ou J, Carbonero F, Zoetendal EG, et al. Diet, microbiota, and microbial metabolites in colon cancer risk in rural Africans and African Americans. Am J Clin Nutr 2013;98:111-20. 
109 de Wit N, Derrien M, Bosch-Vermeulen H, et al. Saturated fat stimulates obesity and hepatic steatosis and affects gut microbiota composition by an enhanced overflow of dietary fat to the distal intestine. Am J Physiol Gastrointest Liver Physiol 2012;303:G589-99.

110 Wan Y, Wang F, Yuan J, et al. Effects of dietary fat on gut microbiota and faecal metabolites, and their relationship with cardiometabolic risk factors: a 6-month randomised controlled-feeding trial. Gut 2019;68:1417-29.

111 Turnbaugh PJ, Ridaura VK, Faith JJ, et al. The effect of diet on the human gut microbiome: a metagenomic analysis in humanized gnotobiotic mice. Sci Trans/ Med 2009;1:6ra14.

112 Cheng M, Ning K. Stereotypes about Enterotype: the old and new ideas. Genomics Proteomics Bioinformatics 2019;17:4-12.

113 Muralidharan J, Moreno-Indias I, Bulló M, et al. Effect on gut microbiota of a 1-y lifestyle intervention with Mediterranean diet compared with energy-reduced Mediterranean diet and physical activity promotion: PREDIMED-Plus study. Am J Clin Nutr 2021;114:1148-1158.

114 Anhê FF, Roy D, Pilon G, et al. A polyphenol-rich cranberry extract protects from diet-induced obesity, insulin resistance and intestinal inflammation in association with increased Akkermansia spp. population in the gut microbiota of mice. Gut 2015;64:872-83

115 Steinert RE, Lee Y-K, Sybesma W. Vitamins for the gut microbiome. Trends Mol Med 2020:26:137-40.

116 Abenavoli L, Scarpellini E, Colica C, et al. Gut microbiota and obesity: a role for probiotics. Nutrients 2019;11. doi:10.3390/nu11112690. [Epub ahead of print: 07 Nov 2019]

117 Slavin J. Fiber and prebiotics: mechanisms and health benefits. Nutrients 2013;5:1417-35.

118 Gibson GR, Beatty ER, Wang $X$, et al. Selective stimulation of bifidobacteria in the human colon by oligofructose and inulin. Gastroenterology 1995;108:975-82.

119 Zhao L, Zhang F, Ding X, et al. Gut bacteria selectively promoted by dietary fibers alleviate type 2 diabetes. Science 2018;359:1151-6.

120 Parker ED, Liu S, Van Horn L, et al. The association of whole grain consumption with incident type 2 diabetes: the Women's Health Initiative Observational Study. Ann Epidemiol 2013;23:321-7.

121 InterAct C, InterAct Consortium. Dietary fibre and incidence of type 2 diabetes in eight European countries: the EPIC-InterAct study and a meta-analysis of prospective studies. Diabetologia 2015;58:1394-408.

122 Müller M, Canfora EE, Blaak EE. Gastrointestinal transit time, glucose homeostasis and metabolic health: modulation by dietary fibers. Nutrients 2018;10. doi:10.3390/ nu10030275. [Epub ahead of print: 28 Feb 2018].

123 Dikeman CL, Fahey GC. Viscosity as related to dietary fiber: a review. Crit Rev Food Sci Nutr 2006;46:649-63.

124 Salonen A, Lahti L, Salojärvi J, et al. Impact of diet and individual variation on intestinal microbiota composition and fermentation products in obese men. Isme J 2014:8:2218-30.

125 Korpela K, Flint HJ, Johnstone AM, et al. Gut microbiota signatures predict host and microbiota responses to dietary interventions in obese individuals. PLoS One 2014:9:e90702.

126 Healey G, Murphy R, Butts C, et al. Habitual dietary fibre intake influences gut microbiota response to an inulin-type fructan prebiotic: a randomised, doubleblind, placebo-controlled, cross-over, human intervention study. Br J Nutr 2018;119:176-89.

127 Rodriguez J, Hiel S, Neyrinck AM, et al. Discovery of the gut microbial signature driving the efficacy of prebiotic intervention in obese patients. Gut 2020;69:1975 1987.

128 Davila A-M, Blachier F, Gotteland M, et al. Intestinal luminal nitrogen metabolism: role of the gut microbiota and consequences for the host. Pharmacol Res 2013;68:95-107.

129 Smith EA, Macfarlane GT. Enumeration of human colonic bacteria producing phenolic and indolic compounds: effects of $\mathrm{pH}$, carbohydrate availability and retention time on dissimilatory aromatic amino acid metabolism. J App/ Bacteriol 1996;81:288-302.

130 Hamer HM, De Preter V, Windey K, et al. Functional analysis of colonic bacterial metabolism: relevant to health? Am J Physiol Gastrointest Liver Physiol 2012;302:G1-9.

131 Scott KP, Gratz SW, Sheridan PO, et al. The influence of diet on the gut microbiota. Pharmacol Res 2013;69:52-60.

132 He L, Han M, Qiao S, et al. Soybean antigen proteins and their intestinal sensitization activities. Curr Protein Pept Sci 2015;16:613-21.

133 Liu Y, Wang Y, Ni Y, et al. Gut microbiome fermentation determines the efficacy of exercise for diabetes prevention. Cell Metab 2020;31:77-91.

134 Canfora EE, Blaak EE. Towards personalized microbial substrates for metabolic health. Nat Rev Endocrinol 2020;16:613-4.

135 Liisberg U, Myrmel LS, Fjære E, et al. The protein source determines the potential of high protein diets to attenuate obesity development in C57BL/6J mice. Adipocyte 2016:5:196-211

136 Fogelholm M, Anderssen S, Gunnarsdottir I, et al. Dietary macronutrients and food consumption as determinants of long-term weight change in adult populations: a systematic literature review. Food Nutr Res 2012;56. doi:10.3402/fnr.v56i0.19103. [Epub ahead of print: 1308 2012]

137 Smith JD, Hou T, Ludwig DS, et al. Changes in intake of protein foods, carbohydrate amount and quality, and long-term weight change: results from 3 prospective cohorts. Am J Clin Nutr 2015;101:1216-24.

138 Mozaffarian D. Dietary and policy priorities for cardiovascular disease, diabetes, and obesity: a comprehensive review. Circulation 2016;133:187-225.

139 Holmes AJ, Chew YV, Colakoglu F, et al. Diet-Microbiome interactions in health are controlled by intestinal nitrogen source constraints. Cell Metab 2017;25:140-51.

140 Ma N, Tian Y, Wu Y, et al. Contributions of the interaction between dietary protein and gut microbiota to intestinal health. Curr Protein Pept Sci 2017;18:795-808.

141 Liu X, Blouin J-M, Santacruz A, et al. High-Protein diet modifies colonic microbiota and luminal environment but not colonocyte metabolism in the rat model: the increased luminal bulk connection. Am J Physiol Gastrointest Liver Physiol 2014;307:G459-70.

142 Walker AW, Ince J, Duncan SH, et al. Dominant and diet-responsive groups of bacteria within the human colonic microbiota. Isme J 2011:5:220-30.

143 Chimerel C, Emery E, Summers DK, et al. Bacterial metabolite indole modulates incretin secretion from intestinal enteroendocrine L cells. Cell Rep 2014;9:1202-8.

144 Coelho OGL, Cândido FG, Alfenas RdeCG. Dietary fat and gut microbiota: mechanisms involved in obesity control. Crit Rev Food Sci Nutr 2019;59:3045-53.

145 Doré J, Blottière $\mathrm{H}$. The influence of diet on the gut microbiota and its consequences for health. Curr Opin Biotechnol 2015;32:195-9.

146 Wolters M, Ahrens J, Romaní-Pérez M, et al. Dietary fat, the gut microbiota, and metabolic health - A systematic review conducted within the MyNewGut project. Clin Nutr 2019;38:2504-20.

147 Laursen MF, Andersen LBB, Michaelsen KF, et al. Infant gut microbiota development is driven by transition to family foods independent of maternal obesity. mSphere 2016;1. doi:10.1128/mSphere.00069-15. [Epub ahead of print: 1002 2016].

148 Danneskiold-Samsoe NB. Dias de Freitas Queiroz Barros H, Santos R, et al. interplay between food and gut microbiota in health and disease. Food Res Int 2019;115:23-31.

149 Sheng L, Jena PK, Liu H-X, et al. Obesity treatment by epigallocatechin-3-gallateregulated bile acid signaling and its enriched Akkermansia muciniphila. Faseb $\mathrm{J}$ 2018:fj201800370R (published Online First: 2018/06/09).

150 Dao MC, Everard A, Aron-Wisnewsky J, et al. Akkermansia muciniphila and improved metabolic health during a dietary intervention in obesity: relationship with gut microbiome richness and ecology. Gut 2016;65:426-36.

151 Depommier C, Everard A, Druart C, et al. Supplementation with Akkermansia muciniphila in overweight and obese human volunteers: a proof-of-concept exploratory study. Nat Med 2019;25:1096-103.

152 Most J, Penders J, Lucchesi M, et al. Gut microbiota composition in relation to the metabolic response to 12-week combined polyphenol supplementation in overweight men and women. Eur J Clin Nutr 2017;71:1040-5.

153 Korem T, Zeevi D, Zmora N, et al. Bread affects clinical parameters and induces gut Microbiome-Associated personal glycemic responses. Cell Metab 2017;25:1243-53.

154 Christensen L, Roager HM, Astrup A, et al. Microbial enterotypes in personalized nutrition and obesity management. Am J Clin Nutr 2018;108:645-51.

155 Hjorth MF, Roager HM, Larsen TM, et al. Pre-Treatment microbial Prevotella-toBacteroides ratio, determines body fat loss success during a 6-month randomized controlled diet intervention. Int J Obes 2018:42:284

156 Muller M, Hermes GDA, Canfora EE. Effect of wheat bran derived prebiotic supplementation on gastrointestinal transit, gut microbiota, and metabolic health: a randomized controlled trial in healthy adults with a slow gut transit. Gut Microbes 2020:1-15 (published Online First: 2020/01/28).

157 Müller M, Hermes GDA, Canfora EE, et al. Distal colonic transit is linked to gut microbiota diversity and microbial fermentation in humans with slow colonic transit. Am J Physiol Gastrointest Liver Physiol 2020:318:G361-9.

158 Most J, Goossens GH, Reijnders D, et al. Gut microbiota composition strongly correlates to peripheral insulin sensitivity in obese men but not in women. Benef Microbes 2017;8:557-62.

159 Blanco-Rojo R, Alcala-Diaz JF, Wopereis S, et al. The insulin resistance phenotype (muscle or liver) interacts with the type of diet to determine changes in disposition index after 2 years of intervention: the CORDIOPREV-DIAB randomised clinical trial. Diabetologia 2016:59:67-76.

160 Guess ND, Dornhorst A, Oliver N, et al. A randomised crossover trial: the effect of inulin on glucose homeostasis in subtypes of prediabetes. Ann Nutr Metab 2016;68:26-34

161 Hermes GDA, Reijnders D, Kootte RS, et al. Individual and cohort-specific gut microbiota patterns associated with tissue-specific insulin sensitivity in overweight and obese males. Sci Rep 2020;10:7523.

162 Mardinoglu A, Wu H, Bjornson E, et al. An integrated understanding of the rapid metabolic benefits of a Carbohydrate-Restricted diet on hepatic steatosis in humans. Cell Metab 2018:27:559-71.

163 Kovatcheva-Datchary P, Nilsson A, Akrami R, et al. Dietary fiber-induced improvement in glucose metabolism is associated with increased abundance of Prevotella. Cell Metab 2015;22:971-82. 
164 Nilsson AC, Johansson-Boll EV, Björck IME. Increased gut hormones and insulin sensitivity index following a 3-D intervention with a barley kernel-based product: a randomised cross-over study in healthy middle-aged subjects. Br J Nutr 2015;114:899-907.

165 Cani PD, Lecourt E, Dewulf EM, et al. Gut microbiota fermentation of prebiotics increases satietogenic and incretin gut peptide production with consequences for appetite sensation and glucose response after a meal. Am J Clin Nutr 2009:90:1236-43.

166 Lobley GE, Holtrop G, Bremner DM, et al. Impact of short term consumption of diets high in either non-starch polysaccharides or resistant starch in comparison with moderate weight loss on indices of insulin sensitivity in subjects with metabolic syndrome. Nutrients 2013:5:2144-72.

167 Robertson MD, Bickerton AS, Dennis AL, et al. Insulin-Sensitizing effects of dietary resistant starch and effects on skeletal muscle and adipose tissue metabolism. Am J Clin Nutr 2005:82:559-67.

168 Dewulf EM, Cani PD, Claus SP, et al. Insight into the prebiotic concept: lessons from an exploratory, double blind intervention study with inulin-type fructans in obese women. Gut 2013;62:1112-21.

169 Vulevic J, Juric A, Tzortzis G, et al. A mixture of trans-galactooligosaccharides reduces markers of metabolic syndrome and modulates the fecal microbiota and immune function of overweight adults. J Nutr 2013;143:324-31. 